\title{
Evaluation of Non-Singular BEM Algorithms for Potential Problems
}

gabriel@dees.ufmg.br

T. S. A. Ribeiro

tatiana_sar@yahoo.com.br

Federal University of Minas Gerais - UFMG

Structural Engineering Department

Belo Horizonte, MG, Brazil

A. B. Jorge

ariosto.b.jorge@unifei.edu.br

Federal University of Itajubá - UNIFEI

Mechanical Engineering Institute

Itajubá, MG, Brazil

T. A. Cruse

tcruse@woh.rr.com

Vanderbilt University

Mechanical Engineering Department

Nashville, TN, USA
Two non-singular boundary element method (BEM) algorithms for two-dimensional potential problems have been implemented using isoparametric quadratic, cubic and quartic elements. The first one is based on the self-regular potential boundary integral equation (BIE) and the second on the self-regular flux-BIE. The flux-BIE requires the $C^{1, \alpha}$ continuity of the density functions, which is not satisfied by the standard isoparametric elements. This requirement is remedied by adopting the relaxed continuity strategy. The self-regular flux-BIE has presented some poor and oscillatory results, mainly with continuous quadratic elements. This odd behavior has completely disappeared when discontinuous elements, which satisfy the continuity requirement, were applied, and this suggests that the 'relaxed continuity hypothesis' seems to be the main cause of numerical errors in the implementation of the self-regular flux-BIE. On the other side, the potential algorithm has shown very reliable solutions.

Keywords: boundary element method, non-singular BEM, self-regular formulations, relaxed continuity, hypersingular formulation

\section{Introduction}

Dealing with singular integrals, including strongly-singular (Cauchy principal value type) and hypersingular (Hadamard finite part type) has been a daunting task in the development of boundary element method (BEM) algorithms. A huge research effort has been devoted to deal with singular integrals, numerically and analytically, in the development of BEM algorithms since the beginning of the method. However, during the last few years, several researchers have demonstrated that the boundary integral equation (BIE) can be remodeled in weakly-singular or non-singular forms, which remove all strongly or hypersingular integrals.

Self-regular forms of the Somigliana displacement identity (SDI) and the Somigliana stress identity (SSI) have been presented by Cruse and Richardson (1996) and Richardson et al. (1997). For 3-D potential theory, Cruse and Richardson (2000) have presented two self-regular formulations of the BIE, while Jorge et al. (2001) in a similar fashion have developed the correspondent self-regular forms for 2-D problems.

The first formulation (potential-BIE) is achieved by subtracting a constant potential from the original BIE, while the second formulation (flux-BIE) is derived by taking the gradient of the potential integral equation. The BIE obtained in this way has one strongly singular and one hypersingular integral. Regularization is then applied to both integrals by means of a linear potential field. Thus, this vector equation is projected in the normal direction, which generates the flux scalar equation. Nevertheless, the higherorder of the singularities in the kernels requires more smoothness of the densities for the given integral to be finite, just like on the standard hypersingular formulation. According to Krishnasamy et al. (1992), a sufficient condition for the existence of the hypersingular integral is the $\mathrm{C}^{1, \alpha}$ continuity of the density function at the source point. Standard isoparametric boundary elements do not satisfy this requirement and for this reason, approximate solution techniques for solving hypersingular integral equations by means of the BEM require special consideration.
In spite of the very successful numerical results reported by Richardson et al. (1997), Richardson and Cruse (1999), Chien et al. (1991), Huang and Cruse (1994) using various forms of these relaxed algorithms combined with piece-wise $C^{1, \alpha}$ interpolations, Martin and Rizzo (1996), Krishnasamy et al. (1992) have concluded that these algorithms could not be theoretically justified. This means that, from a strictly mathematical point of view, only boundary element implementations that ensure $\mathrm{C}^{0, \alpha}$ or $\mathrm{C}^{1, \alpha}$ continuity at each collocation point can be applied in the discretizations of the standard, or the hypersingular boundary integral equations, respectively. Martin et al. (1998) renewed the discussion between the theoretical continuity requirements and the good numerical results reported by Cruse and his co-workers. Jorge et al. (2003) and Porto et al. (2005) presented a non-symmetric variational approach to enforce $C^{1, \alpha}$ continuity requirement at inter-element nodes in the self-regular traction-BIE and flux-BIE, discretized using relaxed continuity approach with Lagrangian $\mathrm{C}^{0}$ elements. They achieved impressive improvements with this approach, mainly when quadratic boundary elements were used, while only small improvements were obtained when the degree of interpolating polynomial was increased to cubic and quartic. The lack of smoothness of the displacement derivatives or potential derivatives at inter-element nodes is shown to be an important source of errors for the traction-BIE and flux-BIE formulation, respectively, especially for quadratic elements.

The main purpose of this work is to investigate and discuss the ability and accuracy of two boundary element method (BEM) algorithms based on the self-regular forms of the potential and the flux boundary integral equations (BIE), respectively. This paper also attempts to answer the claim by Liu and Rudolphi (1999) about the need for a convergence study of the relaxation strategy on the selfregular flux-BIE. The validity of the 'relaxed continuity' hypothesis in the self-regular flux-BIE implementation is investigated by comparing the numerical results of two benchmark problems analyzed with continuous and discontinuous quadratic, cubic and quartic boundary elements. 


\section{Nomenclature}

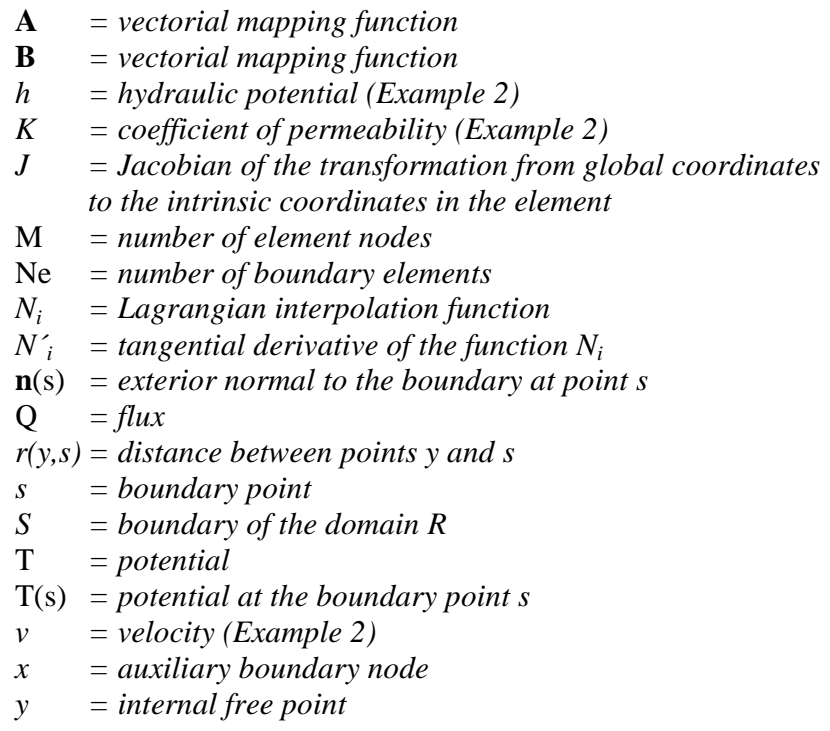

\section{Greek Symbols}

$\nabla h=$ gradient of the hydraulic potential (Example 2)

$\nabla T(s)=$ gradient of the potential at the boundary point $\mathrm{s}$

$\xi=$ intrinsic coordinate in the boundary element

\section{Self-Regular BIE for 2-D Potential Problems}

The process of remodeling the BIE using expressions that contain bounded and easily computable integrals is called regularization. A usual approach for regularization consists in subtracting and adding back certain terms to the integral equations, a process which is equivalent to imposing simple solutions to the BIE. This process is performed so that at the boundary points, where the collocation and the field points coincide, the originally singular integrands would be zero-valued by subtraction, while the remaining added integrals are computable. This regularization process requires that the corresponding density functions satisfy certain continuity requirements, which are the same continuity requirements for the existence of the boundary integrals as representations of the original boundary value problem. In this sense, no extra continuity requirements are added to the equations in order to regularize them. Also, the result of the integrations has a physical meaning and is usually bounded for well-behaved problems. Thus, all the integrals in the BIE are in fact regular from the beginning, although they are not numerically computable in the way they were originally presented. Regularization only shows the natural regular character of these integrals, when the continuity conditions for the densities are satisfied, and thus the process is in fact the one of a selfregularization, the integrals in the BIE having already a self-regular nature, which is brought up by the self-regularization process.

A striking feature about these weakly singular and non-singular BIE formulations is that the two integrals are regularized to weakly singular or non-singular integrals at the same time. The properties of the fundamental solution play an important role in achieving this regularization. Weakly-singular, strongly-singular or hypersingular integrals are cancelled out completely and naturally from both sides of the BIE formulations by exploiting these properties.

In this section these formulations are shown and further details about the analytical development are presented in Jorge et al. (2001) and in Cruse and Richardson (2000). The first formulation is the self-regular potential formulation wherein the unique potential at an arbitrary boundary point is used to regularize the singular integral identity. The self-regular approach emphasizes the fact that the use of singular potentials for Laplace's equation or elasticity is naturally formulated using either bounded or weakly singular potentials. The domain of the potential problem is a finite plane region $R$ bounded by a closed curve $S$ with a outward normal $\mathbf{n}(\mathrm{s})$. The points $(y, s)$ are taken to be the interior free point and the boundary integration point, respectively. The interior form of the Green's identity is given by the following combination of a double and a single layer potential.

$$
\begin{aligned}
& (2 / \pi) T(y)=-\int_{S} T(s) \nabla \ln [1 / r(s, y)] \mathbf{n}(s) d S \\
& +\int_{S} \nabla T(s) \mathbf{n}(s) \ln [1 / r(s, y)] d S \quad \forall y \in R
\end{aligned}
$$

The term $\boldsymbol{\nabla T}(\mathrm{s})$ is the gradient of the potential $T$ evaluated at the boundary point $s$ unless otherwise noted. The potential field $\mathrm{T}(\mathrm{y})$ satisfies Laplace's equation and the boundary conditions for the finite region. Using the directional derivative definition, then

$$
\nabla[\ln (1 / r)] \mathbf{n}(s)=(d / d n) \ln [1 / r(s, y)]
$$

is the derivative of the fundamental solution in the normal direction defined by the outward unit vector $\mathbf{n}(\mathrm{s})$.

The Green's identity for problems in which the potential field is $\mathrm{C}^{0, \alpha}$ continuous in the Hölder sense can be regularized. The procedure of regularization is shown in Jorge et al. (2001) and consists in subtracting and adding back the integral

$$
T(x) \int_{S} \nabla \ln [1 / r(s, y)] \mathbf{n}(s) d S
$$

This integral has the value of $\mathrm{T}(\mathrm{x})(-2 \pi)$, as the swept angle integral of the two-dimensional closed surface is $2 \pi$ for any point $y$ $\in R$. The self-regular form of the potential-BIE is then given by

$$
\begin{aligned}
& (2 / \pi) T(y)=(2 / \pi) T(x)-\int_{S}[T(s)-T(x)] \nabla \ln [1 / r(s, y)] \mathbf{n}(s) d S \\
& +\int_{S} \nabla T(s) \mathbf{n}(s) \ln [1 / r(s, y)] d S
\end{aligned}
$$

The result is the natural form of Green's identity as all unbounded terms are naturally canceled out. The first integral in Eq. (4) is regular and bounded for all points, while the second is weakly singular but also bounded. The result is called self-regularized in that the mathematical properties of the double-layer potential and the Hölder continuity of the harmonic function $T(y)$ are all that is required to modify the original formulation. Since Eq. (4) is continuous for $y \rightarrow x, \forall x \in S$, including at corners, by taking the limit to the boundary, the boundary integral equation is obtained for all boundary points, and is given by

$$
\begin{aligned}
& 0=-\int_{S}[T(s)-T(x))(d / d n) \ln [1 / r(s, y)] d S \\
& +\left.\int_{S}(d T / d n)\right|_{S} \ln [1 / r(s, y)] d S
\end{aligned}
$$

The self-regular form of the flux-BIE is derived by taking the gradient of the integral representation for the potential at the interior point $y$, followed by subtracting and adding back a linear potential field given by

$$
T^{L}(s)=T(x)+\left.T_{, i}\right|_{x}\left[x_{i}(s)-x_{i}(x)\right]
$$


The gradient of this linear field is given by $\nabla \mathrm{T}^{\mathrm{L}}(\mathrm{s})=\nabla \mathrm{T}(\mathrm{x})$. This use of a linear field is the analogous operation for the gradient equation as the constant field is for the regular potential representation. Rudolphi (1991) has presented the same regularization for the hypersingular integral equation by using 'simple solutions' of the potential problem while Muci-Küchler and Rudolphi (1993) have extended the formulation to elastostatics in the same way.

According to the detailed procedure shown in Cruse and Richardson (2000) and Jorge et al. (2001), the following expression is obtained:

$$
\begin{aligned}
& (2 / \pi) T(y)_{, i}=(2 / \pi) T(x)_{, i} \\
& +\int_{S}\left[T(s)-T^{L}(s)\right] \ln [1 / r(s, y)]_{, i j} n_{j}(s) d S \\
& -\int_{S}[\nabla T(s)-\nabla T(x)] \mathbf{n}(s) \ln [1 / r(s, y)]_{, i} d S
\end{aligned}
$$

This equation is the self-regular form of the potential gradient at an interior point $y$, which is regular for all interior point limits to the boundary, where the continuity condition $\mathrm{T}(\mathrm{y}) \in \mathrm{C}^{1, \alpha}$ is satisfied.

By taking the limits to the boundary, $y \rightarrow x$, at all boundary points satisfying the condition $T(y) \in C^{l, \alpha}$, at any given point $s \equiv x$, the following regular boundary integral equation is obtained:

$$
\begin{aligned}
& 0=\int_{S}\left[T(s)-T^{L}(s)\right] \ln [1 / r(s, y)]_{, i j} n_{j}(s) d S \\
& -\int_{S}[\nabla T(s)-\nabla T(x)] \mathbf{n}(s) \ln [1 / r(s, y)]_{, i} d S
\end{aligned}
$$

This vector equation is called the gradient-BIE Green's identity for potential theory. This equation is overspecified and it is usual to operate on it with the local normal $n_{i}(x)$. The resulting scalar equation is called the flux-BIE, and this is the usual scalar form which produces the best numerical results. The flux-BIE is expressed by

$$
\begin{aligned}
& 0=n_{i}(x) \int_{S}\left[T(s)-T^{L}(s)\right] \ln [1 / r(s, y)]_{, i j} n_{j}(s) d S \\
& -n_{i}(x) \int_{S}[\nabla T(s)-\nabla T(x)] \mathbf{n}(s) \ln [1 / r(s, y)]_{, i} d S
\end{aligned}
$$

It is worth noting that the above self-regular formulations, the potential-BIE in Eq. (5) and the flux-BIE in Eq. (9) were obtained through manipulation and limit to the boundary of the integral equations before discretization. The 'self-regular' form of these equations and their limit to the boundary are predicated on the same continuity requirements that went into the derivation of the singular integrals in the original problem, before regularization. Thus, the regularized integrals contain no strong or non-integrable singularity only if the density is sufficiently continuous at the limit as the field point goes to the boundary, as assumed in the derivations. The next section discusses the discretization of these integral equations, where the boundary is discretized into standard isoparametric elements, which satisfy all the continuity requirements for the potential-BIE, but do not satisfy the continuity requirements for the flux-BIE at a finite number of inter-element nodes in case standard continuous isoparametric boundary elements are used. Since these continuity requirements refer only to collocation points, a 'relaxed continuity' approach is introduced for the case of collocation at an inter-element node according to Huang and Cruse (1994), Cruse and Richardson (1996), Richardson et al. (1997), Richardson and Cruse (1999), and different alternatives are discussed, which either allow or avoid the collocation at such nodes. Numerical results obtained from these alternatives, as well as from the self-regular potential BIE are presented.

\section{Computational BEM Algorithms}

The main features of the two non-singular BEM algorithms implemented in this work are described bellow. The first one is based on the self-regular potential-BIE (Eq. (5)) and the second algorithm is based on the self-regular flux-BIE (Eq. (9)). By dividing the boundary $S$ of the domain into $\mathrm{Ne}$ elements, Eq. (5) is rewritten in its discretized form. As in the respective continuous form (Eq. (5)), in the discretized potential-BIE, the first integral, which was originally singular is self-regularized and bounded, while the second remains weakly singular and needs no regularization to be evaluated. Thus, the first integral is numerically evaluated by applying a standard Gaussian quadrature with twelve integration points. Due to the logarithmic nature of the fundamental solution in 2-D, the second integral in Eq. (5) is numerically performed by means of the logarithmic quadrature when the collocation point is placed on the element under integration. Quadratic, cubic and quartic boundary elements based on standard $\mathrm{C}^{0}$ isoparametric representation have been implemented and tested using the potential-BIE formulation.

The BEM algorithm based on the flux-BIE follows the same basic assumptions used for the potential-BIE formulation, but some special features should be emphasized if the same aforementioned interpolation schemes are adopted. These boundary elements do not preserve the $\mathrm{C}^{1, \alpha}$ continuity of the potential that is required for the mathematical validity of the self-regular flux-BIE (Eq. (9)). However, in Richardson et al. (1997) and Richardson and Cruse (1999) the authors claim that this BEM algorithm matches the bounded nature of the BIE in a two-sided sense without invalidating the underlying continuity requirements for the flux formulation, which is the essence of relaxed continuity approach (piecewise smoothness). Unlike the potential-BIE, on the flux-BIE all integrals in Eq. (9) are regular and a standard numerical Gaussian quadrature scheme is used to evaluate these integrals.

The self-regular flux-BIE formulation requires an explicit representation of potential gradient at the boundary. The gradient vector is univocally obtained for each boundary element in terms of the local tangential derivative and the normal derivative (flux). The tangential derivatives of the potential are computed for each boundary element in terms of the intrinsic coordinate $\xi$, according to the following expression:

$$
d T / d S \approx(1 / J) \sum_{i=1}^{m} N_{i}^{\prime}(\xi) T_{i} \quad \text { where } \quad N_{i}^{\prime}=d N_{i} / d \xi
$$

where the functions $\mathrm{N}_{\mathrm{i}}(\xi)$ are the Lagrangian interpolations of order $(m-1)$ in terms of the intrinsic coordinate $\xi, m$ is the number of nodes per element, and the Jacobian $(J)$ is obtained in the usual manner for the isoparametric model of the element geometry. The gradient of the potential at any boundary point is given in terms of mapping functions $\mathbf{A}$ and $\mathbf{B}$, as

$$
\nabla T(s) \approx \nabla T\left(\xi_{s}\right)=\mathbf{A}\left(\xi_{s}\right) \sum_{i=1}^{m} N_{i}^{\prime}(\xi) T^{i}+\mathbf{B}\left(\xi_{s}\right) \sum_{i=1}^{m} N_{i} Q^{i}
$$

where $Q^{j}$ is the nodal value of the flux. The mapping functions $\mathbf{A}$ and $\mathbf{B}$ are given by

$$
\mathbf{A}(\xi)=\left(\begin{array}{c}
-n_{2} / J \\
n_{1} / J
\end{array}\right) \quad \mathbf{B}(\xi)=\left(\begin{array}{l}
n_{1} \\
n_{2}
\end{array}\right)
$$


where $\xi$ and $n_{i}$ are respectively the intrinsic coordinate and the components of the normal unit vector at the integration point $\xi$.

The regularizing terms for a given element, which shares the collocation point $x^{I}$, are expressed as follows:

$$
\begin{aligned}
& T^{L}(s)=T\left(x^{I}\right)+\nabla\left(x^{I}\right)\left(\mathbf{s}^{M}-\mathbf{x}^{I}\right) \\
& \nabla T^{L}(s)=\nabla T\left(x^{I}\right)
\end{aligned}
$$

Therefore, the gradient for the $M$-th element is evaluated using Eq. (11). The potential is continuous and it may be checked that the density functions $T(s)-T^{L}(s)$ and $\nabla T(s)-\nabla T^{L}(s)$ are $O\left[r(s, x)^{2}\right]$ and $O[r(s, x)]$, respectively.

One possible important source of error when using self-regular flux formulation is that due to the evaluation of $\nabla \Gamma(\xi)$ at some point on the boundary, both normal and tangential derivatives of $T(x)$ are required at this point. As the flux-BIE does not explicitly contain the tangential derivative, it is obtained in this code by taking the derivatives of the shape functions that are approximating $T(\xi)$ in an element. Therefore, tangential derivatives in this scheme are obtained from polynomials that are one degree lower than the polynomials used to obtain normal derivatives at the same point. Thus the gradient is obtained in a somewhat unbalanced manner.

On the other hand, the regularizing gradients in Eq. (9) are single-valued in the analytical model at the collocation point $x^{I}$, but these values are not unique in the "relaxed continuity" BEM algorithm. Therefore, the element based values are used locally for integrals in the elements that contain the collocation point, while the average nodal value of the gradient at the collocation is used to regularize the integrals that do not share the collocation point. As a difference exists between the gradients evaluated at any two sharing elements at a node, this causes some error in the solution. Even in a smooth section of the boundary, the origin of this difference is the tangential derivative of the potential, which is written as the derivative of the different interpolating functions, one for each element sharing the collocation point. When the collocation point is placed at an intra-element node or discontinuous elements are used, the regularizing gradient is unique, and this kind of error does not occur because $C^{1, \alpha}$ continuity requirement is met.

In order to investigate which of these sources of error is dominant in this algorithm, discontinuous elements were implemented in the discretization of the self-regular flux-BIE formulation. Such procedure allows the split of the sources of errors introduced by the SRFF. If discontinuous elements are employed, the tangential derivative of the potential is still evaluated through the derivative of the element interpolation function. However, there is no need to adopt the "relaxed continuity" hypothesis, since the smoothness requirement is met at each collocation point, which is always an intra-element node. Therefore, for discontinuous elements, the potential gradient is single valued at each collocation point.

\section{Numerical Results}

\section{Example 1}

A steady state heat conduction problem, called the Motz problem, was analyzed using the BEM algorithms previously explained in the previous section. This is a classical problem in potential theory, used as a benchmark problem for comparison between different approaches to evaluate how accurately they deal with singular fields (Jaswon and Symm, 1977). The geometry and boundary conditions are represented in Fig. 1. There is a singularity at point $\mathrm{O}$ resulting from the existence of a flux discontinuity at this point.

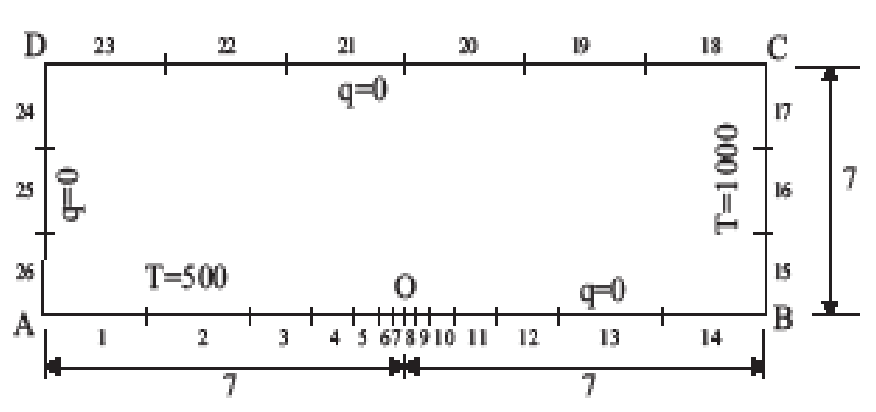

Figure 1. Geometry and boundary conditions of the Motz Problem.

The problem was firstly analyzed using the coarsest mesh shown in Fig. 1, where only the element numbers are represented. A non-hierarchical p-refinement is performed by increasing the order of the interpolation functions by means of the application of quadratic, cubic and quartic boundary elements.

In case discontinuous elements are applied, the collocation points are moved from both ends to positions inside the element at a distance of $5 \%$ of the length of the element.

The potential results on edge OB obtained in this work are compared to the results presented by Jaswon and Symm (1977), París and Cañas (1997) using standard BEM with quadratic boundary elements and Whiteman and Papamichael (1972). The numerical results achieved by the application of the self-regular potential BIE formulation (SRPF) and the results from Jaswon and Symm (1977), París and Cañas (1997) and Whiteman and Papamichael (1972) are in very good agreement, as well as those from the self-regular flux-BIE formulation with discontinuous elements (SRFF-disc.). The maximum errors were $0.44 \%$ for SRPF and $0.09 \%$ for SRFF with discontinuous elements. On the other side, the results from the self-regular flux-BIE with continuous quadratic elements have been shown to be very poor, oscillating around the reference answer. The SRFF results with cubic and quartic continuous elements also have sufficient accuracy.

The flux results obtained by means of the three BEM approaches (SRPF, SRFF, SRFF-disc.) over the edge OA, where it is known that the flux is singular at point $\mathrm{O}$, have shown good agreement to each other for points far enough from the singular point. Again some significant differences have been observed for the SRFF results using continuous quadratic elements. For points very close to the singular point $\mathrm{O}$, the SRPF has presented some very inaccurate results. The results obtained from the SRFF seem to fit the flux behavior better than the potential formulation. Among the flux results for the SRFF approach, the results for discontinuous elements and quartic continuous elements appear to give the best approximation for the singular tendency of the flux at point $O$ as the normalized distance $d / L$ goes to zero.

The poor results using the SRFF combined with continuous quadratic element motivated a convergence study using increasingly refined meshes. Starting from the mesh shown in Fig. 1, seven additional refined meshes were generated by doubling the number of elements consecutively. In each of the straight lines $\mathrm{OA}$ and $\mathrm{OB}$, the ratio of the longest element to the shortest element is 10 , and the grading process follows a geometric progression.

Again, the oscillatory behavior has been observed for all the numerical potential results achieved using the SRFF with continuous quadratic boundary elements for all the graded meshes, while this did not happen in the correspondent answers obtained using quadratic discontinuous elements. The highest numerical errors for each mesh are presented in Fig. 2. 


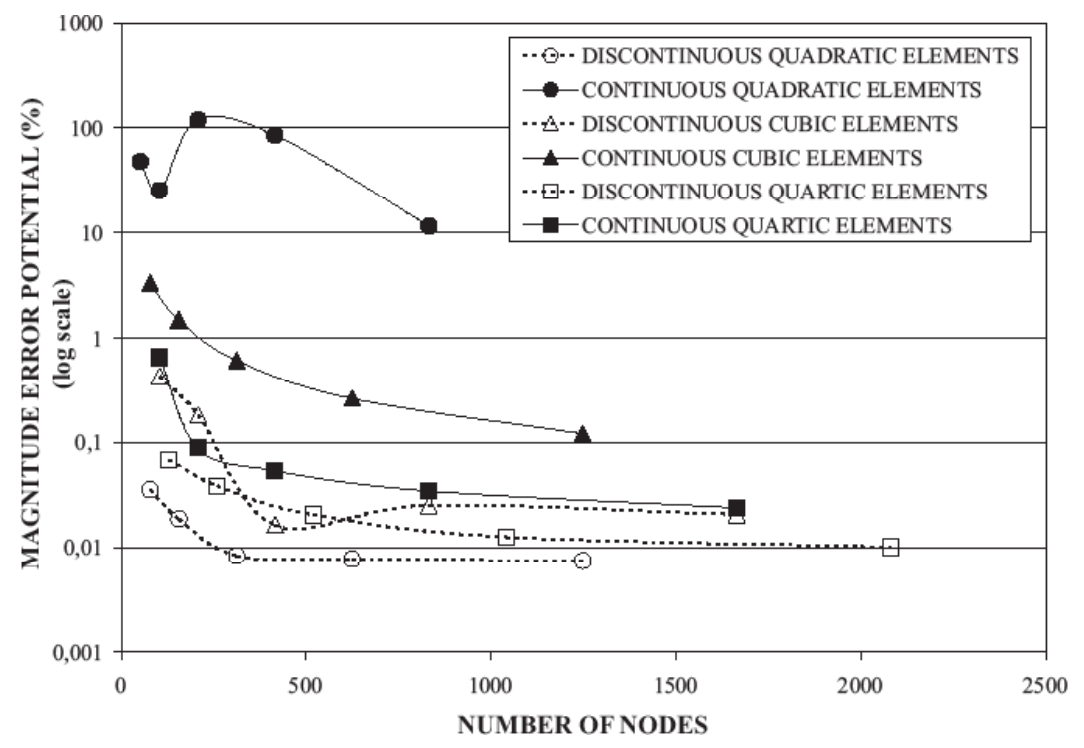

Figure 2. Magnitude error potential in the edge OB using the self-regular flux-BIE.

Analogous behavior is observed for the flux results obtained near to the singular point $\mathrm{O}$ by means of the SRFF with continuous quadratic boundary elements for all the graded refined meshes, which for the sake of brevity are not presented here. As previously pointed out, the poor results for the SRFF with continuous quadratic elements could be related to inaccuracies in the evaluation of the tangential derivative of the potential or the assumption of the "relaxed continuity" hypothesis, implying in non-single valued potential gradient at inter-element nodes. Therefore, in order to investigate the main source of inaccuracies, five meshes with quadratic discontinuous boundary elements similar to meshes R1 to R5 were taken using discontinuous elements instead of continuous elements. Similar meshes with cubic and quartic discontinuous elements were also analyzed. The potential results along the edge OB for the SRFF with discontinuous elements are compared to the solutions presented by Jaswon and Symm (1977). The highest magnitude errors along the edge $\mathrm{OB}$ for both continuous and discontinuous SRFF are shown in Fig. 2.

It can be noticed from Fig. 2 that there is a significant improvement in result accuracy when discontinuous quadratic elements are employed instead of continuous quadratic elements. Moreover, the oscillatory convergence behavior for continuous quadratic elements becomes monotonic for discontinuous elements of the same order. Although there is some improvement in result accuracy when discontinuous cubic and quartic elements are applied, the improvement rate is not as noticeable as for quadratic elements.

It seems that the different improvement rates obtained for quadratic, cubic and quartic interpolations are in someway related with the proportion of inter-element to intra-element nodes. Based on the fact that when adopting the "relaxed continuity" approach the regularizing gradient is not single-valued at inter-element nodes, and that this kind of error is not introduced when the collocation point is placed at an intra-element node in a closed boundary; when using continuous quadratic elements, for each equation related to a collocation point shared by two elements there is an equation related to an intra-element node, which is a proportion of $1: 1$, for cubic elements this proportion is $1: 2$ and in the case of quartic elements $1: 3$. So the analysis using quadratic element is the most critical with regard to regularizing gradient.

\section{Example 2 - Ground Excavation}

The second problem represents the study of the influence of an excavation in the ground water flow according to the Darcy's law, which relates the flux of water through the soil and the gradient of the hydraulic potential. For the isotropic case, this law assumes the form

$$
v=-K \nabla h
$$

where $v$ is the velocity field, $h$ the hydraulic potential and $\mathrm{K}$ the coefficient of permeability. The excavation is large enough to allow a plane study of the ground water flow problem and it is also assumed that there is no influence of the excavation at a distance of $100 \mathrm{~m}$ from its center. The dimensions and boundary conditions are shown in Fig. 3, which also includes the assumed thickness of the dry and wet layers as well as the existence of a waterproof layer on the edge EF. There is an axis of symmetry coincident with the edge AF. Under the hypothesis of incompressible soil and water, this problem is governed by Laplace's equation, with a singularity in flux solution at point $\mathrm{B}$.

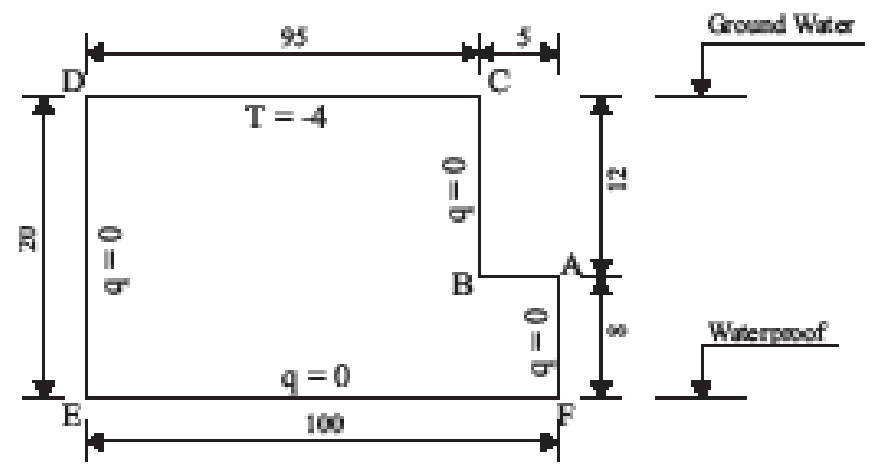

Figure 3. Geometry and boundary conditions - Example 2. 
At first, three different meshes have been analyzed using quadratic, cubic and quartic elements, respectively, and the two selfregular algorithms have been applied. These meshes are referred respectively as M1-quadratic, M1-cubic and M1-quartic, and the boundary points are evenly spaced on all edges except on the edges $\mathrm{AB}$ and $\mathrm{BC}$, where the mesh is graded following a geometric progression, being the ratio of the longest element to the shortest element equal 10.

The numerical results for the potential in the edge $\mathrm{BC}$ using the meshes M1 were compared to finite element (FE) results for a very fine mesh (18432 degrees of freedom) taken as a reference solution, which has been accomplished using ANSYS $®$, version 11.0. The potential results obtained from the SRPF with continuous quadratic, cubic and quartic elements and the SRFF with discontinuous elements and continuous quartic elements are in very close agreement with the FE results and with the numerical solution presented by París and Cañas (1997). Some numerical flux results on the edge $\mathrm{AB}$ are plotted in Fig. 4. On the edge $\mathrm{AB}$, the flux results are very close to each other for points far enough from the singular point $\mathrm{B}$, while near this singular point the local results obtained using the SRPF with all continuous elements and the SRFF algorithm with continuous quadratic and cubic elements are significantly different from the FE results. The results obtained in this example even using coarse meshes show that the SRFF algorithm provides sufficiently accurate results when either discontinuous elements or continuous quartic elements are used.

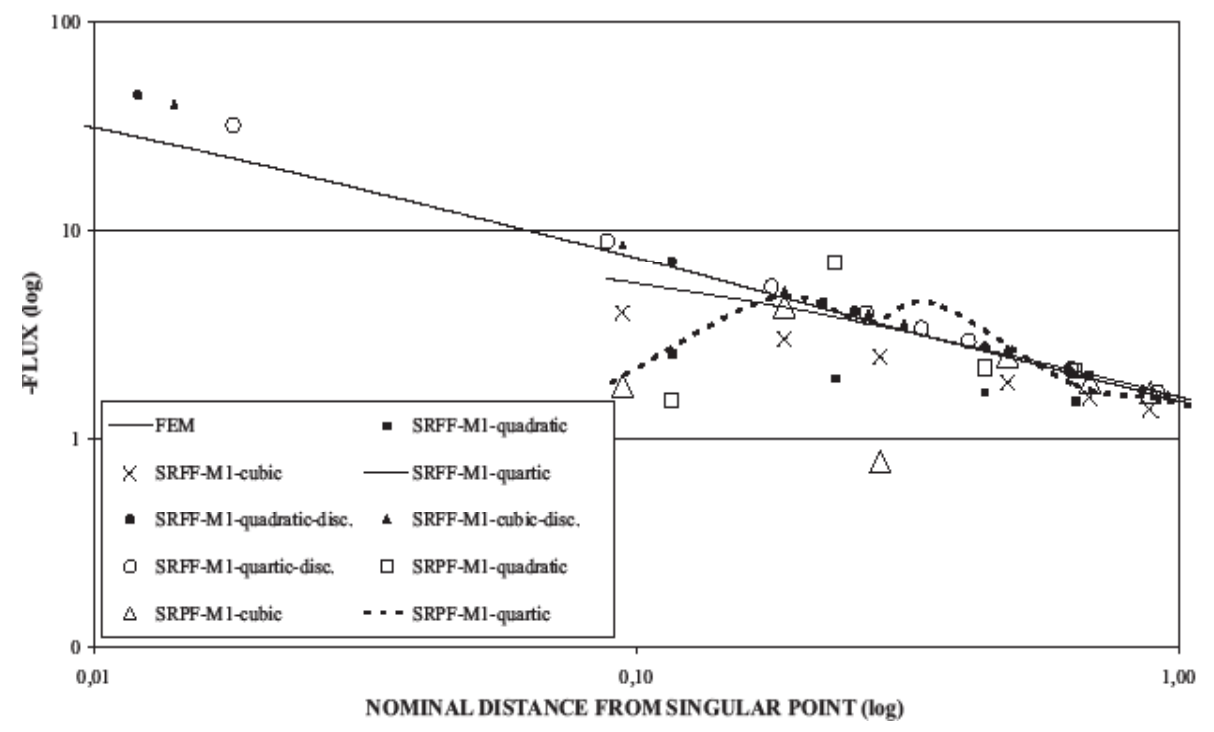

Figure 4. Flux on edge $A B$ near to the singular point.

As can be noticed in both examples, when using SRFF with quadratic elements the results have shown to be very poor and oscillatory. In order to check the convergence of the problem, each of the three basic meshes (M1) has been successively refined splitting each element in two new ones with the same length in all edges except at edges $\mathrm{AB}$ and $\mathrm{BC}$, where the nodes were also relocated according to the geometric progression previously explained for Example 1. Basically the same oscillatory behavior observed in the quadratic-SRFF results of the Example 1 has been observed in Example 2. The possible error introduced by the assumption of the 'relaxed continuity' hypothesis on the self-regular flux formulation was analyzed following the same procedure adopted in Example 1. BEM solutions for potential and flux along the edges $\mathrm{AB}$ and $\mathrm{BC}$, respectively, for meshes M1-M4 using quadratic, cubic and quartic interpolation functions are compared to FE solution to determine the maximum relative error. The results for the magnitude of the error are plotted in Fig. 5 and Fig. 6 for the SRFF with both continuous and discontinuous quadratic, cubic and quartic boundary elements.

Again, it is observed that the accuracy of the results for the SRFF is improved when discontinuous elements are implemented. Also, the improvement rate is higher for quadratic interpolation than for cubic and quartic interpolations. In fact, the use of discontinuous quartic elements on the SRFF algorithm instead of continuous elements of the same order presents the smallest gain in accuracy of the potential and flux.

Based on this example, it seems that the assumption of the 'relaxed continuity' hypothesis has indeed a great influence on the accuracy of the results from the SRFF with continuous elements, despite some good results achieved with quartic elements. The discontinuity of the gradient of the potential has greater influence in results accuracy when cubic and especially quadratic elements are used, since as previously explained, the proportion of inter-element to intra-element nodes is lower in these cases than for quartic elements. These results therefore strengthen the conclusions drawn for the first example.

\section{Conclusions}

The self-regular potential formulation has shown very accurate and stable answers even to treat singular problems. On the other hand, self-regular flux formulation has presented some very poor and oscillatory results, especially when continuous quadratic elements are adopted, even for highly refined meshes. The influence of discontinuity of the regularizing gradient at inter-element nodes, of which value depends upon the element to be integrated, seems to be the main cause, which contributes to some odd results using the SRFF algorithm with continuous boundary elements. Previous work of the authors with the self-regular gradient-based BIE formulations (SRFF and self-regular traction-BIE) for 2-D problems pointed out to the tangential derivative interpolation as the dominant error source, for various degrees of the interpolating functions. The current results show otherwise that the tangential derivatives introduced in this formulation by deriving the interpolation schemes might not be the dominant error source for all cases. 


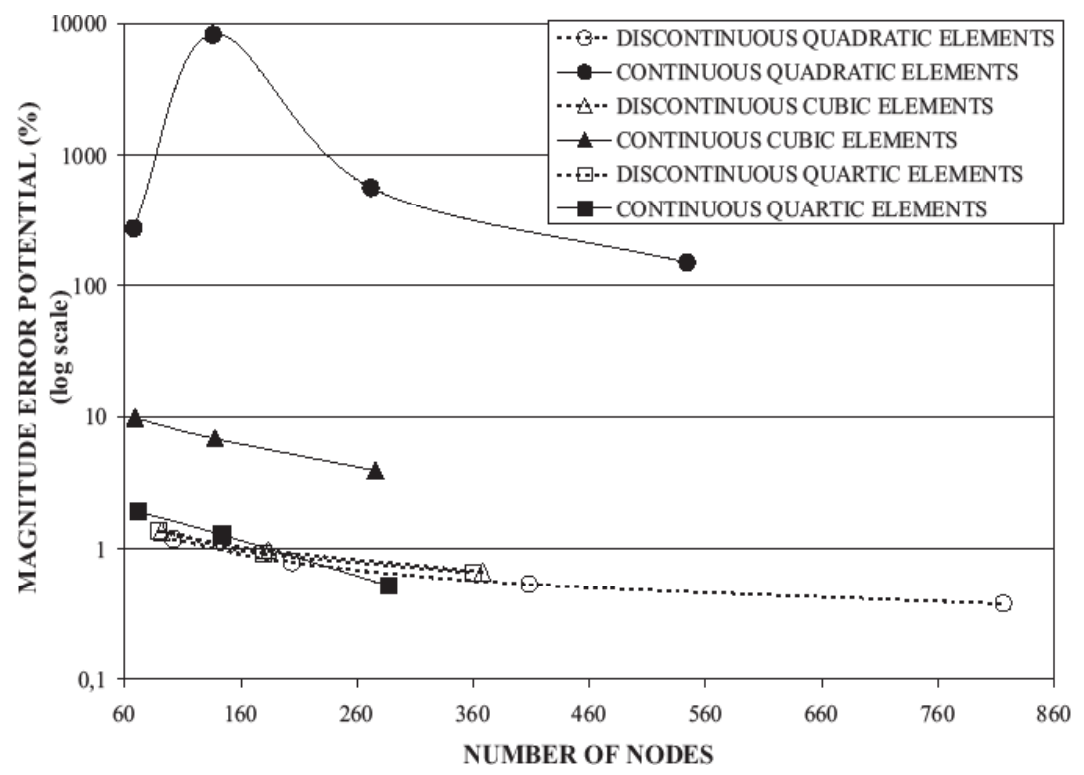

Figure 5. Magnitude error potential using the SRFF.

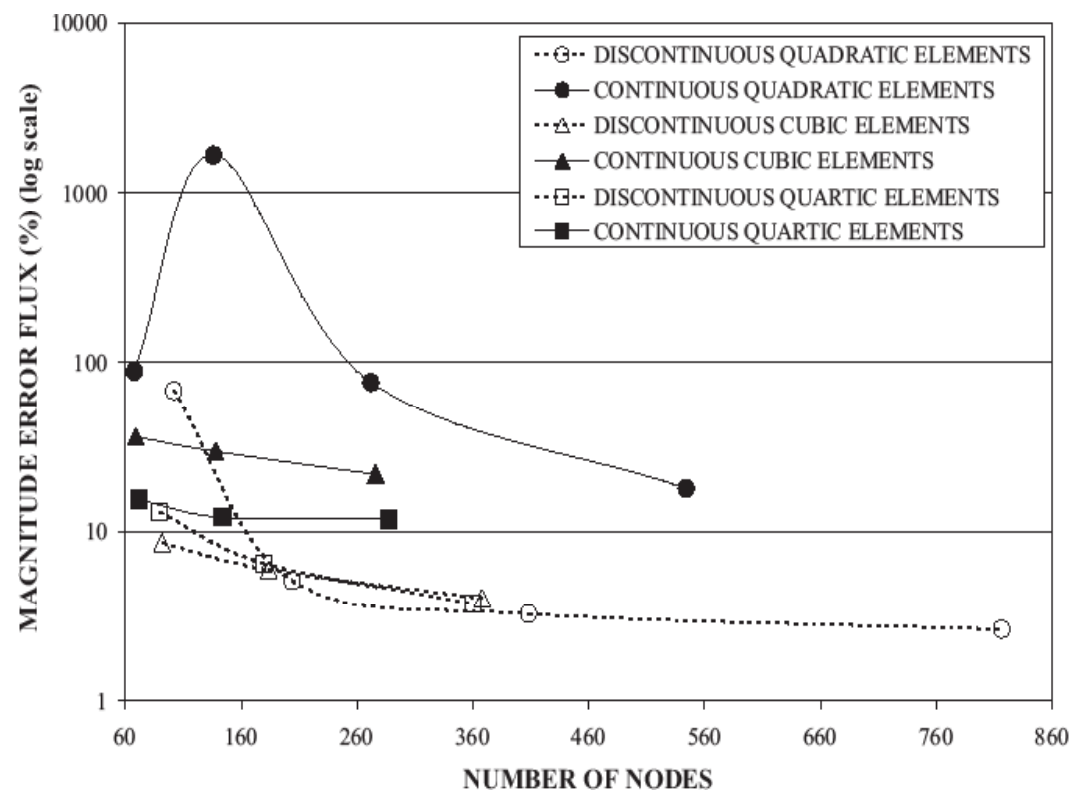

Figure 6. Magnitude error flux using the SRFF.

The explanation for better SRFF results achieved with higher order continuous elements is that when using quadratic elements, for each equation related to an inter-element node there is one equation related to an intra-element node, which represents a proportion of $1: 1$, whereas for cubic and quartic elements this proportion is lower, being $1: 2$ and 1:3 respectively. The oscillatory behavior completely disappeared when the SRFF with discontinuous elements have been applied and the BEM solutions are very accurate. In this case, all collocation points are restricted to intra-element nodes where the densities are always $C^{1, \alpha}$ Hölder continuous. The comparison between the SRFF results based on continuous elements and the correspondent ones obtained by means of discontinuous elements leads to conclusion that the relaxed continuity is not reliable and that the smoothness requirements assumed in the derivation of the BIE formulations must be preserved. This kind of error does not happen when the source point is located at an intra-element node, where the smoothness requirement is met and the present numerical results clearly bring this fact out. Although the use of standard continuous elements is more appealing and easier than continuous elements based on $\mathrm{C}^{1}$ interpolation functions or even discontinuous elements, from the results obtained so far, it seems that the use of this kind of element on the SRFF is not reliable. It seems that the smoothness requirement for the SRFF should be satisfied in order to guarantee results accuracy and convergence. 


\section{Acknowledgements}

The authors gratefully acknowledge the support received from the following Brazilian agencies of research funding: $\mathrm{CNPq}-$ Conselho Nacional de Desenvolvimento Científico e Tecnológico; FAPEMIG - Fundação de Amparo à Pesquisa do Estado de Minas Gerais and CAPES - Coordenação de Aperfeiçoamento de Pessoal de Nível Superior.

\section{References}

Chien, C. C., Rajiyah, H. and Atluri, S. N., 1991, "On the evaluation of hyper-singular integrals arising in the boundary element method for linear elasticity", Computational Mechanics, Vol. 8, pp. 57-70.

Cruse, T. A. and Richardson, J. D, 1996, " Non-singular somigliana stress identity in elasticity", International Journal for Numerical Methods in Engineering, Vol. 39, pp. 3273-3304.

Cruse, T. A. and Richardson, J. D., 2000, "Self-regularized Hypersingular BEM for Laplace's Equation", In: Matemathical Aspects of Boundary Element Methods, Ed. Chapman \& Hall, Boca Raton.

Huang, Q. and Cruse, T. A., 1994, "On the non-singular traction-BIE in elasticity", International Journal for Numerical Methods in Engineering, Vol. 37, pp. 2041-2072.

Jaswon, M. A. and Symm, G. T., 1977, "Integral Equation Methods in Potential Theory and Elastostatics", Ed. Academic Press.

Jorge, A. B., Cruse, T. A., Fisher, T. S. and Ribeiro, G. O., 2003, “A new variational self-regular traction-BEM formulation for inter-element continuity of displacement derivatives", Computational Mechanics, Vol. 32, pp. 401-414.

Jorge, A. B., Ribeiro, G. O., Cruse, T. A. and Fisher, T. S., 2001, "Selfregular boundary integral equation formulations for Laplace's equation in 2D", International Journal for Numerical Methods in Engineering, Vol. 51, pp.1-29.
Krishnasamy, G., Rizzo, F. J. and Rudolphi, T. J., 1992, "Continuity requirements for density funcions in the boundary integral equation method", Computational Mechanics, Vol. 9, pp. 267-284.

Liu, Y. J. and Rudolphi, T. J., 1999, "New identities for fundamental solutions and their applications to nonsingular boundary element formulations", Computational Mechanics, Vol. 24, pp. 286-292.

Martin, P. A. and Rizzo, F. J., 1996, "Hypersingular integrals: how smooth must the density be?", International Journal for Numerical Methods in Engineering, Vol. 39, pp. 687-704.

Martin, P. A., Rizzo, F. J. and Cruse, T. A., 1998, "Smoothnessrelaxation strategies for singular and hypersingular equations", International Journal for Numerical Methods in Engineering, Vol. 42, pp. 885-906.

Muci-Küchler, K. H. and Rudolphi, T. J., 1993, "Coincident collocation of displacement and tangent derivative boundary integral equations in elasticity", International Journal for Numerical Methods in Engineering, Vol. 36, pp. 2837-2849.

París, F. and Cañas, J., 1997, "Boundary Element Method: Fundamentals and Applications", Oxford University Press.

Porto, P. A. C., Jorge, A. B. and Ribeiro, G. O., 2005, "Extension of the variational self-regular approach for the flux boundary element method formulation", Computer Modeling in Engineering \& Sciences, Vol. 10, pp. $65-77$.

Richardson, J. D. and Cruse, T. A., 1999, "Weakly singular stress-BEM for 2D elastostatics", International Journal for Numerical Methods in Engineering, Vol. 45, pp. 13-35.

Richardson, J. D., Cruse, T. A. and Huang, Q., 1997, "On the validity of conforming BEM algorithms for hypersingular boundary integral equations", Computational Mechanics, Vol. 20, pp. 213-220.

Rudolphi, T. J., 1991, "Use of simple solutions in the regularization of hypersingular boundary integral equations", Mathematical Computer Modelling, Vol. 15, pp. 269-278.

Whiteman, J. R. and Papamichael, N., 1972, "Treatment of mixed boundary value problems by confromal transformation methods", Journal of Applied Mathematics and Physics, Vol. 23, pp. 655-664. 